\title{
Lattice QCD calculation using VPP500
}

\author{
Seyong $\mathrm{Kim}^{\mathrm{a*}}$ and Shigemi Ohta ${ }^{\mathrm{b} \dagger}$
}

aHEP Division, Argonne National Laboratory, 9700 South Cass Avenue, Argonne, Illinois 60439, USA

bInstitute of Physical and Chemical Research (RIKEN), Wako-shi, Saitama 351-01, Japan

A new vector parallel supercomputer, Fujitsu VPP500, was installed at RIKEN earlier this year. It consists of 30 rector computers, each with 1.6 GFLOPS peak speed and $256 \mathrm{MB}$ memory, connected by a crossbar switch with $400 \mathrm{MB} / \mathrm{s}$ peak data transfer rate each way between any pair of nodes. The authors developed a Fortran lattice QCD simulation code for it. It runs at about 1.1 GFLOPS sustained per node for Metropolis pure-gauge update, and about 0.8 GFLOPS sustained per node for conjugate gradient inversion of staggered fermion matrix.

\section{INTRODUCTION}

A new super-computing system was installed at Institute of Physical and Chemical Research (RIKEN) at the end of March, 1994. It serves various researches in physics, chemistry and biology at RIKEN, and a significant amount of its cpu time is allotted for numerical calculations in lattice quantum chromodynamics (QCD).

\section{HARDWARE}

The core of this system is a new "vector parallel" supercomputer, Fujitsu VPP500. It consists of two main-frame cpu's and 30 "processor elements (PE's)", all connected by a crossbar switch. In addition, there are a $8 \mathrm{~GB}$ semiconductor disk, $40 \mathrm{~GB}$ magnetic disk to store the system and user files, $60 \mathrm{~GB}$ RAID3 and $80 \mathrm{~GB}$ RAID7 disks for data storage, and $10 \mathrm{~TB}$ tape archive.

One of the main-frame cpu's acts as a front-end for the computer. The other, the "control processor (CP)", controls I/O between the PE's and the front-end, disks, and other peripheral devices. It has a $100 \mathrm{MHz}$ scalar cpu with LIW architecture and $128 \mathrm{MB}$ memory. The cpu is made of GaAs and ECL chips and the nemory consists of $1 \mathrm{Mbit}$ SRAMs with 18 ns laiency.

\footnotetext{
- S.K. is supported by the L.S. Department of Energy, Division of High Energy Physics, Contract W-31-109-ENG38. He aiso thanks RIKE. for its support in the early stage of this work.

Talk presented by S.O
}

A single PE consists of the same $100 \mathrm{MHz}$ scalar cpu as the $C P$, a vector processor with 1.6 GFLOPS peak speed, and $256 \mathrm{MB}$ memory. The processors are again made of GaAs and ECL chips. The scalar cpu has 32 32-bit general purpose registers and 3264 bit floating-point registers. The vector processor has $128 \mathrm{~KB}$ vector registers, $2 \mathrm{~KB}$ mask registers, and pipelines for multiplication, add/logic, division, mask, load and store operations. All the floating point operations are done in IEEE 64-bit format. The memory consists of the same kind of SRAMs as in the CP.

The crossbar switch is again a combination of GaAs and ECL chips. It provides data communication at the rate of $400 \mathrm{MB} / \mathrm{s}$ each way between any pair of PE's. It also provides data transfer to the outside through the $8 \mathrm{~GB}$ semiconductor disk connected to the $\mathrm{CP}$, at more than $50 \mathrm{MB} / \mathrm{s}$ to the fastest RAID3 disk, about $20 \mathrm{MB} / \mathrm{s}$ to the $40 \mathrm{~GB}$ system disk, about $10 \mathrm{MB} / \mathrm{s}$ to the $80 \mathrm{~GB}$ RAID7, and a few MB/s to the tape archive which is connected through another crossbar switch that connects the entire super-computing system to the local area network.

\section{SYSTEM SOFTWARE}

The front-end is run by an Unix SVR4 system. The CP and PE's are run by modified Unix systems with enhancement for communications through the crossbar switch. This enhancement 


\section{DISCLAIMER}

Portions of this document may be illegible in electronic image products. Images are produced from the best available original document. 
allows arbitrary partitioning of the PE's. At the moment the 30 PE's are divided into 28-PE and 2-PE partitions during the night, and $16-\mathrm{PE}, 8-$ $P E, 4-P E$ and 2-PE partitions during the day.

Users edit their application programs on the front-end which is reachable from within the local area network. Compilation of the programs and production run are done only in the batch mode handled by a modified NQS systems running in cooperation on both the front-end and CP.

Three different programming languages, Fortran, C, and assembler, are provided. Fortran is based on the Fortran 77 standard with enhancement of vector and parallel directives as comment lines. C is based on its ANSI standard with vector and parallel directives as pragma statements. A vectorized numerical processing library is available for both languages. $\mathrm{C}$ is also augmented by $\mathrm{P} 4$ and PVM message passing libraries.

\section{BENCHMARK TESTS}

Procurement of this super-computing system started in late April, 1993, and took until the middle of December that year. Among the various benchmark tests in the process, the following are worthy to note: a) LINPACK speed, without any restriction for the array sizes, exceeded 30 GFLOPS. b) A $\mathrm{CR}(1)$ conjugate residual solver for staggered quarks of lattice QCD on a $32^{4}$ lattice runs at more than 30 GFLOPS with 28 PE's. c) A fast Fourie: transformation program for onedimensional data of real numbers runs at about 20 GFLOPS for up to $2 \mathrm{~GB}$ of data.

\section{INSTALLATION}

The hardware installation was completed by the end of March, 1994, and test operations started immediately. By the end of April singlePE operations stabilized. Unfortunately parallel operations at fist had some software problems, especially for larger partitions, but finally stabilized by the end of July. The system has been running quite well since then.

\section{LATTICE QCD CALCULATION}

We use Fortran for lattice QCD coding because it is more efficient than $\mathrm{C}$ at the moment. In Fortran, parallel directives are given by comment lines starting with "! xocl".

PE configuration: the parallel directive allows from one-dimensional to seven-dimensional configuration of the available PE's,

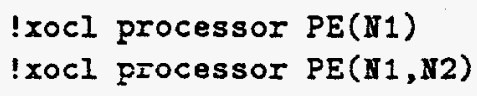

where $11, N 2, \ldots$, denote the number of PE's in each dimension. The first example shows how to allocate $\mathbb{N} 1$ PE's configured on a one-dimensional line segment, while the second shows $\$ 1 \times \mathbb{Z}$ PE's configured on a rectangle, etc. Since we have only $30 \mathrm{PE}$ 's at most, we are practically limited to one-, two-, three- or four-dimensional configuration. We use the one-dimensional for simplicity.

Arrays: we define how to distribute array data by the following directives and definitions,

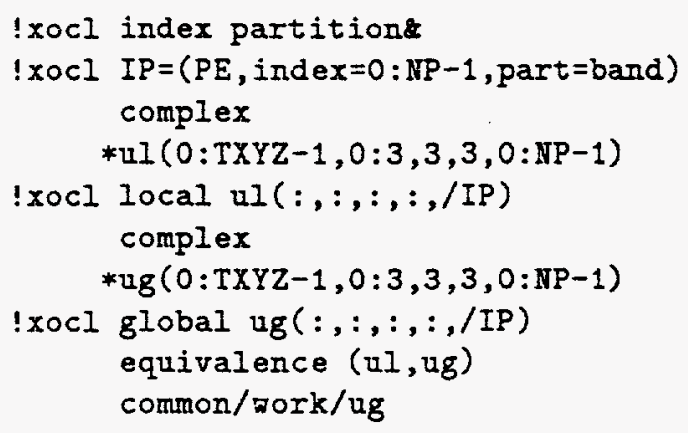

An "index partition", IP, defines that any array index running from 0 through $M P-1$ is distributed over the ITP PE's configured in one dimension. The symbol " $\&$ " means the xocl directive is continued to the next line. Here a TXYZ $\times$ $4 \times 9$ byte contiguous partition of a "local" partitioned array $u l$ is stored on each PE. A local partitioned array is very quickly accessed by the local processors, but cannot be accessed from nonlocal ones. A "global" partitioned array can be accessed by either local or nonlocal processors, but less quickly. As a result of the equivalence statement, the local partitioned array $u I$ and the global one ug share the same memory area on each PE. We use local partitioned arrays for 
most of the calculations and global ones for only inter-PE communications. The common statement is used for sub-procedure interface because a partitioned array name cannot appear as an argument of sub-procedures.

Data structure: again we tried from onethrough four-dimensional mapping of the lattice. One-dimensional mapping gives too heavy load for data communication. Three- and fourdimensional ones are not flexible enough in changing the lattice size. We use two-dimensional mapping. Since we use one-dimensional PE configuration, we explicitly calculate the two-dimensional $\mathrm{PE}$ coordinates, say $\mathrm{n} 1$ and $\mathrm{n} 2$, from the onedimensional $P E$ identification number $n 0,0 \leq n 0$ $\leq \mathbb{M P}-1$, like $\mathrm{nO}=\mathrm{n} 1+\mathrm{MZ} * \mathrm{n} 2$.

Data transfer: we move around some data from one $\mathrm{PE}$ to another using a combination of parallel directives "spread do" and "spread move",

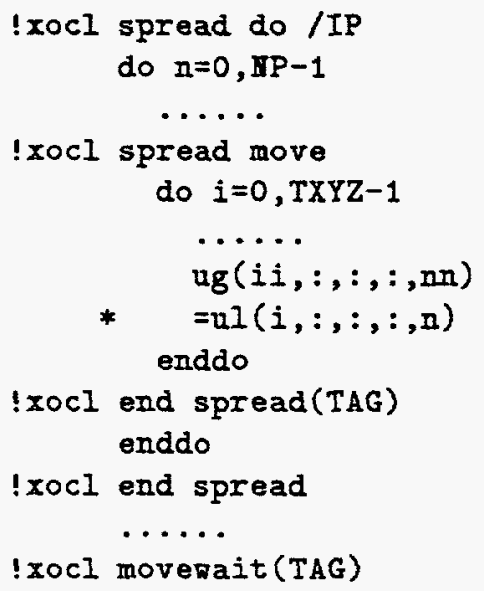

Here nn defines a PE to which the data are sent from the PE $n$, while ii defines a nonlocal array coordinate as a function of the local coordinate $i$. The "end spread(TAG)" directive means the data have been sent out of the local PE, but may not have reached the destination yet. The other directive "movewait(TAG)" tells the PE to wait until the sent-out data have been completely stored in the memory of the destination PE. In between these two directives each PE is free to do other tasks such as those do not affect or are not affected by the data being transferred. This way we can hide the cost of data transfer behind numerical calculations. Actual data transfer speed reaches about $330 \mathrm{MB} / \mathrm{s}$ sustained per $\mathrm{PE}$ one way, out of $400 \mathrm{MB} / \mathrm{s}$ peak, if we transfer more than a couple of $\mathrm{MB}$ at a time which is common for lattice QCD.

Numerical calculations are distributed to the PE's easily by the "spread do" directive:

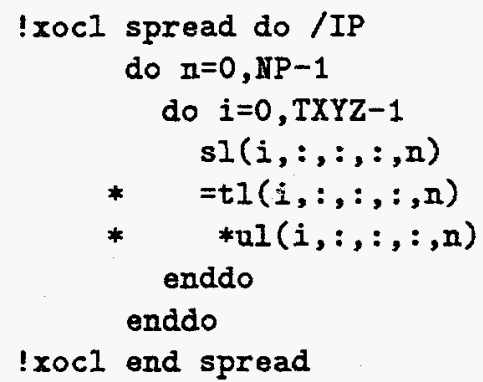

By using suitable buffer arrays, most of the calculations are done using only local partitioned array. Such calculations for $\mathrm{SU}(3)$ matrix and vector operations can easily reach a speed of more than 1.3 GFLOPS sustained per PE out of the 1.6 GFLOPS peak. This is achieved by the Fortran compiler alone and does not require any assembler-level optimization by hand.

Unfortunately, the inter-PE data transfer and local calculations interfere with each other in accessing the memory. This interference often reduces the overall efficiency to about 1.0 GF per $P E$. The interference decreases if we use higher dimensional mapping of the lattice than the current two-dimensional one.

Our test runs on the 8-PE partition of the machine for a $64 \times 16^{3}$ lattice achieved the speed of about $6 \mu$ s per Metropolis link update per PE and $25 \mathrm{~ms}$ per CG iteration for the entire lattice. These numbers should translate into about 1.1 and 0.8 GFLOPS sustained respectively. We are preparing for production runs on larger lattices using partitions with up to $28 \mathrm{PE}$ 's [1]. It should be faster by up to $20 \%$. We do not see any problem in moving up to still larger partitions, like the ones with $64 \mathrm{PE}$ 's or more planned at KEK, since the crossbar switch has been proved to work for as many as $128 \mathrm{PE}$ 's.

\section{REFERENCES}

1. S. Kim and S. Ohta, in preparation. 